\title{
The vasopeptidase inhibitor AVE7688 ameliorates Type 2 diabetic nephropathy
}

\author{
S. Schäfer ${ }^{1}$, W. Linz ${ }^{1}$, H. Vollert ${ }^{1}$, G. Biemer-Daub ${ }^{2}$, H. Rütten ${ }^{1}$, M. Bleich ${ }^{1}$, A. E. Busch ${ }^{1}$ \\ ${ }^{1}$ Disease Group Cardiovascular Diseases, Aventis Pharma, Deutschland GmbH, Frankfurt am Main, Germany \\ ${ }^{2}$ Clinical Chemistry, Aventis Pharma Deutschland GmbH, Frankfurt am Main, Germany
}

\section{Abstract}

Aim/hypothesis. Pharmacological inhibition of the renin angiotensin system has proven clinical efficacy in nephropathies of various origins, including diabetic nephropathy. We tested the effects of the dual inhibition of both angiotensin converting enzyme and neutral endopeptidase by the vasopeptidase inhibitor AVE7688 in an animal model of Type 2 diabetic nephropathy.

Methods. We treated 56 obese Zucker diabetic fatty (ZDF, Gmi-fa/fa) rats aged 34-weeks with either placebo $(n=9)$ or the vasopeptidase inhibitor AVE7688 in four different doses (each $n=9 ; 3,10,30$, or $60 \mathrm{mg} / \mathrm{kg} / \mathrm{d}$ in chow). We used 11 heterozygous (+/fa) rats which received placebo and served as non-diabetic, lean controls. Urinary albumin/creatinine ratio was assessed as a marker of nephropathy at baseline (age 34-weeks) and after 10 weeks of chronic treatment.

Results. All obese animals had established diabetes mellitus that was not influenced by AVE7688 $\left(\mathrm{HbA}_{1 \mathrm{c}}\right.$
$>12 \%$, stable in all dose groups). There was massive albuminuria in the homozygous ZDF rats (albumine/creatinine ratio $>20 \mathrm{mg} / \mathrm{mg}$ vs minimal albuminuria in lean controls) that was decreased by AVE7688 in a dose dependent manner (Placebo 2.0 44.4 vs $11.9 \pm 1.8,13.4 \pm 0.7,13.6 \pm 2.8$, and $19.8 \pm 2.8 \mathrm{mg} / \mathrm{mg}$ in the $3,10,30$, and $60 \mathrm{mg} / \mathrm{kg} / \mathrm{d}$ groups, respectively; all treatment groups $p<0.05$ vs Placebo).

Conclusion/interpretation. AVE7688 ameliorates proteinuria in Zucker diabetic fatty rats with established diabetes mellitus. Vasopeptidase inhibition represents an effective novel therapeutic principle for intervention in Type 2 diabetic nephropathy independent of metabolic control. [Diabetologia (2004) 47:98-103]

Keywords Zucker diabetic fatty rat - diabetes - microvascular complications - diabetic nephropathy · albuminuria - angiotensin converting enzyme - neutral endopeptidase - vasopeptidase inhibition - AVE7688
As a result of its dramatically increasing incidence, Type 2 diabetes has become the leading single cause of end stage renal disease in most industrialized countries [1]. Urinary excretion of albumin has been estab-

Received: 26 February 2003 / Revised: 8 August 2003

Published online: 14 November 2003

C) Springer-Verlag 2003

S. Schäfer ( Disease Group Cardiovascular Diseases, Aventis Pharma, Deutschland GmbH, Building H 821, 65926 Frankfurt am Main, Germany

E-mail: stefan.schaefer@aventis.com

Abbreviations: ACE, angiotensin converting enzyme; ZDF, Zucker diabetic fatty. lished as a sensitive marker of the degree of diabetic kidney damage, and the extent of albuminuria reflects the risk of cardiovascular end points as well as future incidence of end stage renal failure [2]. Pharmacological blockade of the renin angiotensin aldosterone system by either inhibition of angiotensin converting enzyme (ACE) or blockade of the angiotensin II receptor has clinically proven its ability to delay the progression of diabetic nephropathy $[3,4,5,6,7,8]$. The novel vasopeptidase inhibitors could be of therapeutic value in a variety of nephropathies, based on their potential to increase tissue concentrations of bradykinin and the organoprotective natriuretic peptides through inhibition of neutral endopeptidase in addition 
to ACE [9]. Indeed, the vasopeptidase inhibitor omapatrilat has shown potent nephroprotection in models of subtotal rat nephrectomy $[10,11]$ and in a hypertensive, hypo-insulinaemic nephropathy model [12]. However, the potential benefit of pharmacological intervention with a vasopeptidase inhibition in insulin resistant, Type 2 diabetic nephropathy has not been evaluated so far.

In this study, we therefore tested whether chronic treatment with AVE7688, a novel vasopeptidase inhibitor, can effectively reduce urinary albumin excretion, an established biomarker of renal dysfunction, in diabetic nephropathy. We used the Zucker diabetic fatty (ZDF) rat as a suitable model because it is characterized by obesity, insulin resistance, Type 2 diabetes, and progressive proteinuria.

\section{Materials and methods}

Compounds. AVE7688 and ramipril are manufactured by Aventis Pharma Deutschland (Frankfurt am Main, Germany) AVE7688 is a potent simultaneous inhibitor of two peptidases, ACE and neutral endopeptidase 24.11. The chemical name of AVE7688 is 7-[[(2S)-2-(acetylthio)-1-oxo-3-methylpropyl]amino]-1,2,3,4,6,7,8,12b-octahydro-6-oxo-(4S,7S, 12bR)-pyrido[2,1-a][2]benzapin-4-carboxy-acid, with a molecular weight of 432.54 (Fig. 1). In vitro, half maximal inhibitory concentrations $\left(\mathrm{IC}_{50} \mathrm{~s}\right)$ were determined as $0.053 \mathrm{nmol} / \mathrm{l}$ for $\mathrm{ACE}$ and $5.0 \mathrm{nmol} / \mathrm{l}$ for neutral endopeptidase [13]. An in vitro selectivity screen revealed no relevant binding (less than $25 \%$ at $10 \mu \mathrm{mol} / \mathrm{l})$ to a large variety of receptors.

Animals. The animal experiments were carried out in accordance with the Aventis Laboratory Animal Science and Welfare (LASW) guidelines and the German law for the protection of animals. The "Principles of laboratory animal care" (NIH publication no. 85-23, revised 1985) were followed.

Male Zucker diabetic fatty rats (ZDF/Gmi-fa/fa) and their heterozygous lean littermates (ZDF/Gmi-+/fa) were purchased from Charles River Germany (Sulzfeld, Germany) and housed in the local LASW facilities in Frankfurt-Hoechst. The animals were housed individually in standard cages and received a

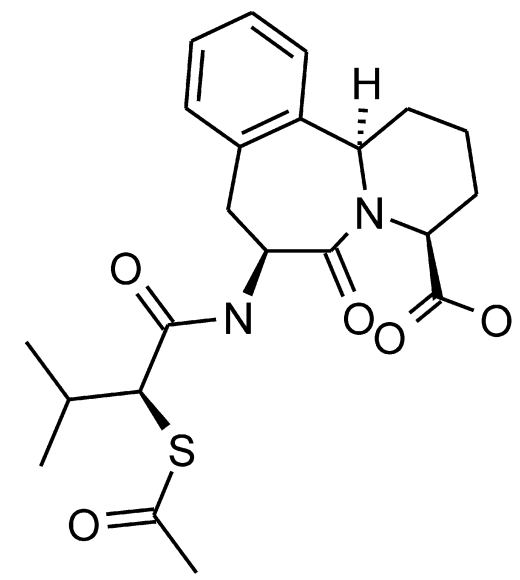

Fig. 1. Chemical structure of AVE7688 standard chow diet (standard diet \#1320, Altromin, Lage, Germany) and tap water ad libitum.

Acute pharmacodynamics. In preliminary studies, we assessed the ability of AVE7688 to inhibit serum ACE activity. Normal Wistar rats weighing 250 to $350 \mathrm{~g}$ were treated by single dose gavage. AVE7688 was dissolved in $1 \mathrm{ml}$ hydroxy-ethyl cellulose and given to normal Wistar rats (250 to $350 \mathrm{~g}$ ) per gavage at different concentrations ranging from 0.01 to $1.0 \mathrm{mg} / \mathrm{kg}$ (each dose group, $n=4$ ). The rats were anaesthetized $20 \mathrm{~min}$ later with pentobarbital $(50 \mathrm{mg} / \mathrm{kg}$ i.p.) and artificially ventilated. Blood samples were taken via a polyethylene catheter from the carotid artery at $45 \mathrm{~min}, 2 \mathrm{~h}$, and $4 \mathrm{~h}$ after administration. Thereafter, the animals were killed under ongoing anaesthesia.

Haemodynamics. The haemodynamic effects of AVE7688 were measured after chronic treatment with two representative doses. Homozygous, diabetic ZDF rats aged 36 weeks were treated with either placebo or AVE7688 at 3 or $30 \mathrm{mg} / \mathrm{kg} / \mathrm{d}$ in chow (each group $n=6$ ). After 6 weeks, heart rate and systemic blood pressure were measured using the tail cuff method; 20 heterozygous, lean animals served as controls.

Albuminuria study. We randomly assigned $56 \mathrm{ZDF}$ rats aged 34 weeks to either of five groups: the Placebo group did not receive specific treatment. The other four groups (each $n=9$ ) received AVE7688 pressed into standard Altromin chow at concentrations of $30 \mathrm{ppm}, 100 \mathrm{ppm}, 300 \mathrm{ppm}$, or 600 ppm. Taking into account an approximate body weight of $370 \mathrm{~g}$ and a daily food intake of approximately $50 \mathrm{~g}$ these concentrations in chow correspond to expositions of $3,10,30$, and $60 \mathrm{mg}$ AVE7688/kg/d, respectively. The 11 heterozygous, lean rats serving as non-diabetic controls (LEAN) received standard chow. All animals received Enrofloxacin (Baytril, Bayer, Leverkusen, Germany; $2.5 \%$ in drinking water) as a prophylaxis against urinary tract infections. Body weight as well as food and water intake over $24 \mathrm{~h}$ were determined at the beginning of the study (i.e., at age 34-weeks) and after 10 weeks of chronic therapy. At these time points, blood was taken from the tail for determination of $\mathrm{HbA}_{1 \mathrm{c}}$. Simultaneously, random urine samples were collected.

Laboratory measurements. Creatinine (in urine and serum) and $\mathrm{HbA}_{1 \mathrm{c}}$ (in whole blood) were determined using standard kits (Roche diagnostics, Basel, Switzerland) on a Hitachi 912 E analyzer; (Hitachi, Mountain View, Calif., USA). Urinary albumin was determined using a fluorescence dye binding assay (Mikroflural, Progen Biotechnik, Heidelberg, Germany) and normalized by urinary creatinine concentration. For determination of ACE activity, the hydrolysis of the tripeptide N-[3-2(furyl)acryloyl]-L-phenylalanyl-glycyl-glycin (FAPPG) into furylacryloyl-phenylalanine and glycine-glycine was measured spectrophotometrically as the decrease in absorption at $\lambda=$ $340 \mathrm{~nm}$ in comparison to known standards. Serum ACE activity was always measured freshly on the day of the experiment, without intermittent freezing. All other serum and urine samples were frozen and stored at $-20^{\circ} \mathrm{C}$ until the day of analysis when necessary.

Statistical analysis. Differences between groups were tested using unpaired Student's $t$-tests, corrected for multiple according to Bonferroni-Holm. Due to the clear distinction in the major outcome parameters between lean and obese animals, differences were not tested against the lean control group. Data are given as means \pm SEM. A $p$ value of less than 0.05 was considered statistically significant. 
Table 1. Metabolic parameters

\begin{tabular}{|c|c|c|c|c|c|c|}
\hline & \multirow[t]{2}{*}{ Lean control } & \multicolumn{5}{|l|}{ ZDF obese } \\
\hline & & Placebo & AVE 3 & AVE 10 & AVE 30 & AVE 60 \\
\hline 34 weeks & $437 \pm 5$ & $368 \pm 10$ & $366 \pm 8$ & $370 \pm 6$ & $372 \pm 8$ & $387 \pm 9$ \\
\hline 44 weeks & $463 \pm 5$ & $353 \pm 8$ & $364 \pm 9$ & $369 \pm 8$ & $370 \pm 10$ & $386 \pm 8$ \\
\hline 34 weeks & $21 \pm 1$ & $47 \pm 1$ & $46 \pm 2$ & $48 \pm 1$ & $48 \pm 2$ & $49 \pm 1$ \\
\hline \multirow[t]{2}{*}{44 weeks } & $23 \pm 0.4$ & $48 \pm 2$ & $49 \pm 3$ & $48 \pm 3$ & $51 \pm 2$ & $51 \pm 1$ \\
\hline & Water intake, & & & & & \\
\hline 34 weeks & $24 \pm 2$ & $213 \pm 7$ & $211 \pm 10$ & $219 \pm 9$ & $225 \pm 10$ & $232 \pm 8$ \\
\hline 44 weeks & $43 \pm 8$ & $214 \pm 9$ & $221 \pm 15$ & $233 \pm 22$ & $235 \pm 12$ & $244 \pm 8$ \\
\hline
\end{tabular}

Values are means \pm SEM. $n=9$ (ZDF obese groups), $n=11$ (Lean controls). AVE, chronic treatment with AVE7688 in chow. The numbers after "AVE" refer to the daily average dose $(\mathrm{mg} / \mathrm{kg} / \mathrm{d})$

Table 2. Urinary creatinine and albumin excretion

\begin{tabular}{|c|c|c|c|c|c|c|}
\hline & \multirow[t]{2}{*}{ Lean control } & \multicolumn{5}{|l|}{ ZDF obese } \\
\hline & & Placebo & AVE 3 & AVE 10 & AVE 30 & AVE 60 \\
\hline 34 weeks & $71 \pm 7$ & $772 \pm 126$ & $1175 \pm 218$ & $953 \pm 114$ & $891 \pm 55$ & $1436 \pm 275$ \\
\hline \multirow[t]{2}{*}{44 weeks } & $86 \pm 17$ & $1321 \pm 327$ & $1448 \pm 405$ & $725 \pm 157$ & $625 \pm 135$ & $634 \pm 122 *$ \\
\hline & \multicolumn{6}{|c|}{ Urinary creatinine concentration, $\mathrm{mmol} / \mathrm{l}$} \\
\hline 34 weeks & $9.63 \pm 0.70$ & $0.30 \pm 0.03$ & $0.44 \pm 0.06$ & $0.40 \pm 0.03$ & $0.36 \pm 0.02$ & $0.45 \pm 0.05$ \\
\hline \multirow[t]{2}{*}{44 weeks } & $13.06 \pm 1.02 *$ & $0.62 \pm 0.06 *$ & $0.83 \pm 0.18 *$ & $0.69 \pm 0.05 *$ & $0.61 \pm 0.04 *$ & $0.62 \pm 0.06 *$ \\
\hline & \multicolumn{6}{|c|}{ Urinary albumin/creatinine ratio, $\mathrm{mg} / \mathrm{mg}$} \\
\hline 34 weeks & $0.07 \pm 0.01$ & $22.7 \pm 3.6$ & $24.5 \pm 3.0$ & $21.3 \pm 2.0$ & $22.7 \pm 1.9$ & $29.0 \pm 3.9$ \\
\hline 44 weeks & $0.06 \pm 0.01$ & $20.7 \pm 5.9$ & $14.7 \pm 2.2 *$ & $9.0 \pm 1.8 *$ & $9.1 \pm 1.9 *$ & $9.2 \pm 1.9 *$ \\
\hline
\end{tabular}

Values are means \pm SEM. $n=9$ (ZDF obese groups), $n=11$ (Lean controls). AVE, chronic treatment with AVE7688 in chow. The numbers after "AVE" refer to the daily average dose (mg/kg/d). * $p<0.05$ vs 34 weeks

\section{Results}

Acute pharmacodynamics. AVE7688 reduced serum ACE activity in a dose dependent manner. At $120 \mathrm{~min}$ after gavage, ACE activity was reduced from $160 \pm 9 \mathrm{U} / 1$ (placebo) to $53 \pm 4,25 \pm 3,22 \pm 4,19 \pm 1$, and $11 \pm 1$ after $0.01,0.03,0.1,0.3$, and $1.0 \mathrm{mg} / \mathrm{kg}$, respectively (all $p<0.05$ vs placebo). The ACE activities at the time points $45 \mathrm{~min}$ and $240 \mathrm{~min}$ were not different from the 120 min values (details not shown).

Haemodynamics study. Heart rate was $351 \pm 11,311 \pm 8$, $321 \pm 11$, and $312 \pm 7 \mathrm{bpm}$ in the lean, ZDF placebo, AVE $3 \mathrm{mg} / \mathrm{kg} / \mathrm{d}$ and AVE $30 \mathrm{mg} / \mathrm{kg} / \mathrm{d}$ groups, respectively. These values were not different from each oth- er. Systolic blood pressure was $154 \pm 3,156 \pm 3,152 \pm 3$, and $143 \pm 5 \mathrm{mmHg}$ in the lean, ZDF placebo, AVE $3 \mathrm{mg} / \mathrm{kg} / \mathrm{d}$, and AVE $30 \mathrm{mg} / \mathrm{kg} / \mathrm{d}$ groups, respectively. These values were not different from each other.

Albuminuria study. Fundamental and metabolic data at weeks 34 and 44 are given in Table 1. Obese animals had established diabetes mellitus, as manifested by excessive food and water intake and substantially increased $\mathrm{HbA}_{1 \mathrm{c}}$ values, compared to lean controls. Of note, AVE7688 did not influence metabolic control, as evidenced by the lack of effect on $\mathrm{HbA}_{1 \mathrm{c}}$ and on food intake.

Data on urinary creatinine and albumin are given in Table 2. Urinary albumin was markedly increased in 


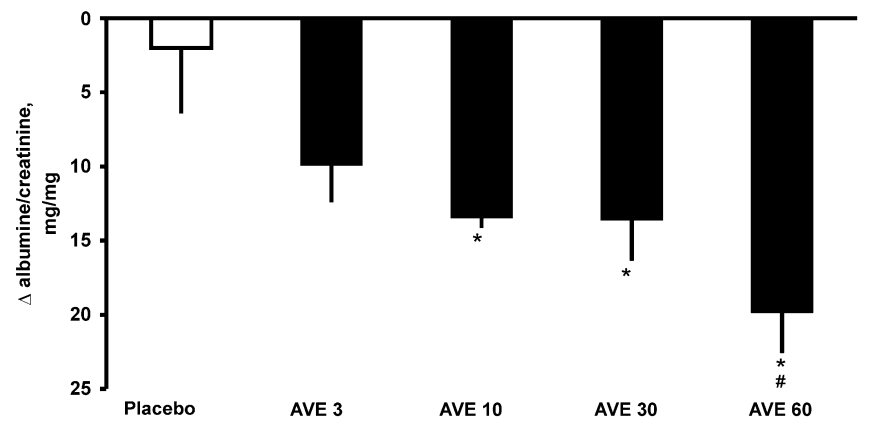

Fig. 2. Decrease in albuminuria produced by different daily doses of AVE7688 in ZDF rats. There is a dose dependent decrease in albumin/creatinine ratio by chronic treatment for 10 weeks, starting at age 34 -weeks. ${ }^{*} p<0.05$ vs Placebo; ${ }^{\#} p<0.05$ vs AVE $3 \mathrm{mg} / \mathrm{kg} / \mathrm{d}$

all obese ZDF rats, compared to lean controls, whether expressed as a crude concentration or normalized to urinary creatinine. After 10 weeks of treatment, the urinary albumin/creatinine ratio was reduced in all dose groups. This reduction was approximately $66 \%$ in the $60 \mathrm{mg} / \mathrm{kg} / \mathrm{d}$ group and greater compared to the $3 \mathrm{mg} / \mathrm{kg} / \mathrm{d}$ group $(p<0.05)$. The differences in albu$\mathrm{min} /$ creatinine ratio between weeks 34 and 44 are illustrated in Fig. 2.

\section{Discussion}

Diabetic nephropathy and albuminuria in the ZDF rat. Diabetic nephropathy is expected to remain the leading cause of end stage renal failure in the Western world, despite the recent therapeutic advances brought about by the angiotensin receptor blockers. Albuminuria is an early and easily measurable biomarker of nephropathy [14]. The extent of albuminuria correlates with the risk of future cardiovascular events (myocardial infarction, stroke) and predicts the incidence of end stage renal failure even before retention parameters such as creatinine are increased in the serum $[2,15]$. For these reasons, we used urinary albumin excretion as a validated marker of nephropathy in this study. In the ZDF (Gmi-fa/fa) rat, the development of nephropathy is characterized by progressive albuminuria and histopathological changes resembling human diabetic nephropathy [13, 16]. Interestingly, there seems to be a transient period of glomerular hyperfiltration (measured as an increased creatinine clearance at age 27 -weeks ) in the ZDF rat, which is very similar to the natural time course of diabetic nephropathy in humans. However, serum creatinine levels are not increased until age 37 weeks [13]. Thus, the ZDF rat model reflects a relatively early clinical stage of diabetic nephropathy.

Effect of vasopeptidase inhibition. Diabetic nephropathy is the result of a complex interplay between meta- bolic and haemodynamic factors [17]. Recent studies show that optimization of glycaemic and blood pressure control can reduce microvascular complications of diabetes by up to 50\% [18]. Anti-hypertensive agents interrupting the renin angiotensin system have been particularly effective in retarding the progression of diabetic nephropathy $[3,4,6]$. Based on its potent and specific simultaneous inhibition of both ACE and neutral endopeptidase, AVE7688 represents a novel class of pharmacological compounds, the vasopeptidase inhibitors [9]. The decrease in serum ACE activity, observed in our study, shows that AVE7688 is a potent inhibitor of ACE also in vivo. Unfortunately, we have not been able to measure the activity of neutral endopeptidase, as there is no reliable assay available so far. However, the prototype vasopeptidase inhibitor, omapatrilat, which has similar in vitro potency as AVE7688 [9], has proven superior efficacy over pure ACE inhibitors in a variety of models $[11,19]$. Future studies will show whether AVE7688, too, is superior to pure ACE inhibition and whether this is mediated through its inhibition of neutral endopeptidase.

Our data provide evidence that vasopeptidase inhibition can ameliorate diabetic nephropathy, even if treatment is started when diabetes and concomitant nephropathy are already established. The dose dependency of this effect indicates that the nephroprotection is related to the mechanism of action of the compound. The extent of the anti-proteinuric effect (up to $66 \%$ reduction) is substantial. For comparison, single therapy in advanced diabetic nephropathy has reduced proteinuria by approximately $30 \%$ in clinical trials $[4$, 6]. Combination therapy using an ACE inhibitor plus angiotensin receptor blocker has recently shown to decrease proteinuria in non-diabetic nephropathy by $75 \%$ [20]. It remains to be investigated whether the addition of an angiotensin receptor blocker to AVE7688 can reduce proteinuria even further.

AVE7688 did not influence glycaemic control in this study, as proven by stable $\mathrm{HbA}_{1 \mathrm{c}}$ values. Inhibition of ACE could be able to ameliorate insulin resistance and, consequently, reduce the incidence of diabetes mellitus. This has been shown clinically with the ACE inhibitor ramipril in the HOPE trial [21] and more recently with the vasopeptidase inhibitor omapatrilat in an experimental study in young Zucker rats [19]. It is important to note that in our study treatment was started at a relatively late stage, when diabetes was already fully established. The absence of an effect on $\mathrm{HbA}_{1 \mathrm{c}}$ therefore does not exclude an effect on insulin sensitivity. The potential insulin sensitizing effects of AVE7688 need to be explored in future studies, using experimental settings specifically suitable to assess insulin resistance.

In the ZDF rat, the blood pressure lowering effects of AVE7688 seem to be minimal, which is in contrast to the potent antihypertensive effects of AVE7688 [22] and other vasopeptidase inhibitors [12, 23, 24, 
25 ] in humans and in different rat models. A possible explanation for the absence of a relevant blood pressure effect is the fact that the ZDF rat is not a hypertensive animal model. Interestingly, the angiotensin receptor blocker losartan does not protect from nephropathy in the Zucker rat despite a reduction in blood pressure [26]. The dual mode of action, particularly the effect on kinin metabolism in addition to intervention in the renin angiotensin system, could explain the dramatic efficacy of the vasopeptidase inhibitor AVE7688 in this model of Type 2 diabetic nephropathy. In a previous study, chronic treatment with an effective dose of AVE7688 (45 mg/kg/d) had no influence on creatinine clearance when the rats were older than 27 weeks [13]. We believe, therefore, that the anti-proteinuric effects of AVE7688 are not mediated via relevant decreases in the glomerular filtration rate in the ZDF rat, although we did not measure serum creatinine in the present study. Increasing evidence suggests a crucial role of the intra-renal renin-angiotensin system in diabetic nephropathy [27]. In contrast, vasopeptidase inhibition seems to afford superior protection from diabetic nephropathy compared to pure ACE inhibition [12, 13]. These observations suggest a substantial role of kinin metabolism in the pathogenesis of diabetic nephropathy. However, the relative importance of neutral endopeptidase compared with ACE for the must be clarified in future studies The vasopeptidase inhibitor AVE7688 provided effective nephroprotection in the absence of metabolic effects and major systemic blood pressure effects. It therefore seems that local renal mechanisms account for a major portion of the anti-albuminuric effects of AVE7688. If this mode of action can be confirmed in clinical studies, the vasopeptidase inhibitors will be able to support the pharmacological armentarium for the treatment of diabetic nephropathy beyond established therapeutic principles such as blood pressure normalization, glucose control, and lifestyle modifications.

Acknowledgements. The authors wish to thank G. Fischer and P. Hainz for excellent technical support. The present study is part of the "Cardiovascular and Renal Endpoints in Diabetes (CARED)" pre-clinical study program at Aventis Pharma.

\section{References}

1. Ismail N, Becker B, Strzelczyk P, Ritz E (1999) Renal disease and hypertension in non-insulin-dependent diabetes mellitus. Kidney Int 55:1-28

2. Opie LH, Parving HH (2002) Diabetic nephropathy: can renoprotection be extrapolated to cardiovascular protection? Circulation 106:643-645

3. Lewis EJ, Hunsicker LG, Bain RP, Rohde RD (1993) The effect of angiotensin-converting-enzyme inhibition on diabetic nephropathy. The Collaborative Study Group. N Engl J Med 329:1456-1462
4. Lewis EJ, Hunsicker LG, Clarke WR et al. (2001) Renoprotective effect of the angiotensin-receptor antagonist irbesartan in patients with nephropathy due to type 2 diabetes. N Engl J Med 345:851-860

5. Wright JT Jr, Bakris G, Greene T et al. (2002) Effect of blood pressure lowering and antihypertensive drug class on progression of hypertensive kidney disease: results from the AASK trial. JAMA 288:2421-2431

6. Brenner BM, Cooper ME, Zeeuw D de et al. (2001) Effects of losartan on renal and cardiovascular outcomes in patients with type 2 diabetes and nephropathy. N Engl J Med 345:861-869

7. Parving HH, Lehnert H, Brochner-Mortensen J, Gomis R, Andersen S, Arner P (2001) The effect of irbesartan on the development of diabetic nephropathy in patients with type 2 diabetes. N Engl J Med 345:870-878

8. Viberti G, Wheeldon NM (2002) Microalbuminuria reduction with valsartan in patients with type 2 diabetes mellitus: a blood pressure-independent effect. Circulation 106:672678

9. Molinaro G, Rouleau JL, Adam A (2002) Vasopeptidase inhibitors: a new class of dual zinc metallopeptidase inhibitors for cardiorenal therapeutics. Curr Opin Pharmacol $2: 131-141$

10. Cao Z, Burrell LM, Tikkanen I, Bonnet F, Cooper ME, Gilbert RE (2001) Vasopeptidase inhibition attenuates the progression of renal injury in subtotal nephrectomized rats. Kidney Int 60:715-721

11. Taal MW, Nenov VD, Wong W et al. (2001) Vasopeptidase inhibition affords greater renoprotection than angiotensinconverting enzyme inhibition alone. J Am Soc Nephrol 12:2051-2059

12. Davis BJ, Johnston CI, Burrell LM et al. (2003) Renoprotective effects of vasopeptidase inhibition in an experimental model of diabetic nephropathy. Diabetologia 46:961971

13. Schäfer S, Linz W, Bube A et al. (2003) Vasopeptidase inhibition prevents nephropathy in Zucker diabetic fatty rats. Cardiovasc Res 60:447-454

14. Caramori ML, Fioretto P, Mauer M (2000) The need for early predictors of diabetic nephropathy risk: is albumin excretion rate sufficient? Diabetes 49:1399-1408

15. Cooper ME (1998) Pathogenesis, prevention, and treatment of diabetic nephropathy. Lancet 352:213-219

16. Hoshi S, Shu Y, Yoshida F et al. (2002) Podocyte injury promotes progressive nephropathy in Zucker diabetic fatty rats. Lab Invest 82:25-35

17. Cooper ME (2001) Interaction of metabolic and haemodynamic factors in mediating experimental diabetic nephropathy. Diabetologia 44:1957-1972

18. Gaede P, Vedel P, Larsen N, Jensen GV, Parving $\mathrm{HH}$, Pedersen O (2003) Multifactorial intervention and cardiovascular disease in patients with type 2 diabetes. $\mathrm{N}$ Engl J Med 348:383-393

19. Wang CH, Leung N, Lapointe N et al. (2003) Vasopeptidase inhibitor omapatrilat induces profound insulin sensitization and increases myocardial glucose uptake in Zucker fatty rats: Studies comparing a vasopeptidase inhibitor, angiotensin-converting enzyme inhibitor, and angiotensin II type I receptor blocker. Circulation 107:19231929

20. Nakao N, Yoshimura A, Morita H, Takada M, Kayano T, Ideura T (2003) Combination treatment of angiotensin-2 receptor blocker and angiotensin-converting-enzyme inhibitor in non-diabetic renal disease (COOPERATE): a randomised controlled trial. Lancet 361:117-124 
21. Heart Outcomes Prevention Evaluation Study Investigators (2000) Effects of ramipril on cardiovascular and microvascular outcomes in people with diabetes mellitus: results of the HOPE study and MICRO-HOPE substudy. Lancet 355:253-259

22. Schäfer S, Linz W, Afkam F et al. (2003) Treatment of hypertensive nephropathy by the novel vasopeptidase inhibitor AVE7688: Nephroprotection beyond ACE inhibition alone. Nephrol Dial Transplant 18[suppl 4]:601 (Abstract)

23. Regamey F, Maillard M, Nussberger J, Brunner HR, Burnier M (2002) Renal hemodynamic and natriuretic effects of concomitant Angiotensin-converting enzyme and neutral endopeptidase inhibition in men. Hypertension 40:266-272

24. Rodriguez-Gomez I, Wangensteen R, Atucha NM et al. (2003) Effects of omapatrilat on blood pressure and renal injury in L-NAME and L-NAME plus DOCA-treated rats. Am J Hypertens 16:33-38

25. Rossi GP, Cavallin M, Rizzoni D et al. (2002) Dual ACE and NEP inhibitor MDL-100,240 prevents and regresses severe angiotensin II-dependent hypertension partially through bradykinin type 2 receptor. J Hypertens 20:14511459

26. Crary GS, Swan SK, O’Donnell MP, Kasiske BL, Katz SA, Keane WF (1995) The angiotensin II receptor antagonist losartan reduces blood pressure but not renal injury in obese Zucker rats. J Am Soc Nephrol 6:1295-1299

27. Carey RM and Siragy HM (2003) The intrarenal reninangiotensin system and diabetic nephropathy. Trends Endocrinol Metab 14:274-281 